\title{
Duplexing mode, ARB and modulation approaches parameters affection on LTE uplink waveform
}

\author{
Fatima Faydhe Al-Azzawi ${ }^{1}$, Faeza Abas Abid ${ }^{2}$, Zainab Faydhe Al-Azzawi ${ }^{3}$ \\ ${ }^{1,2}$ Electronic Technical Department, Institute of Technology, Middle Technical University, Iraq \\ ${ }^{3}$ Department Computer Technology Engineering AL- Mansour University College, Iraq
}

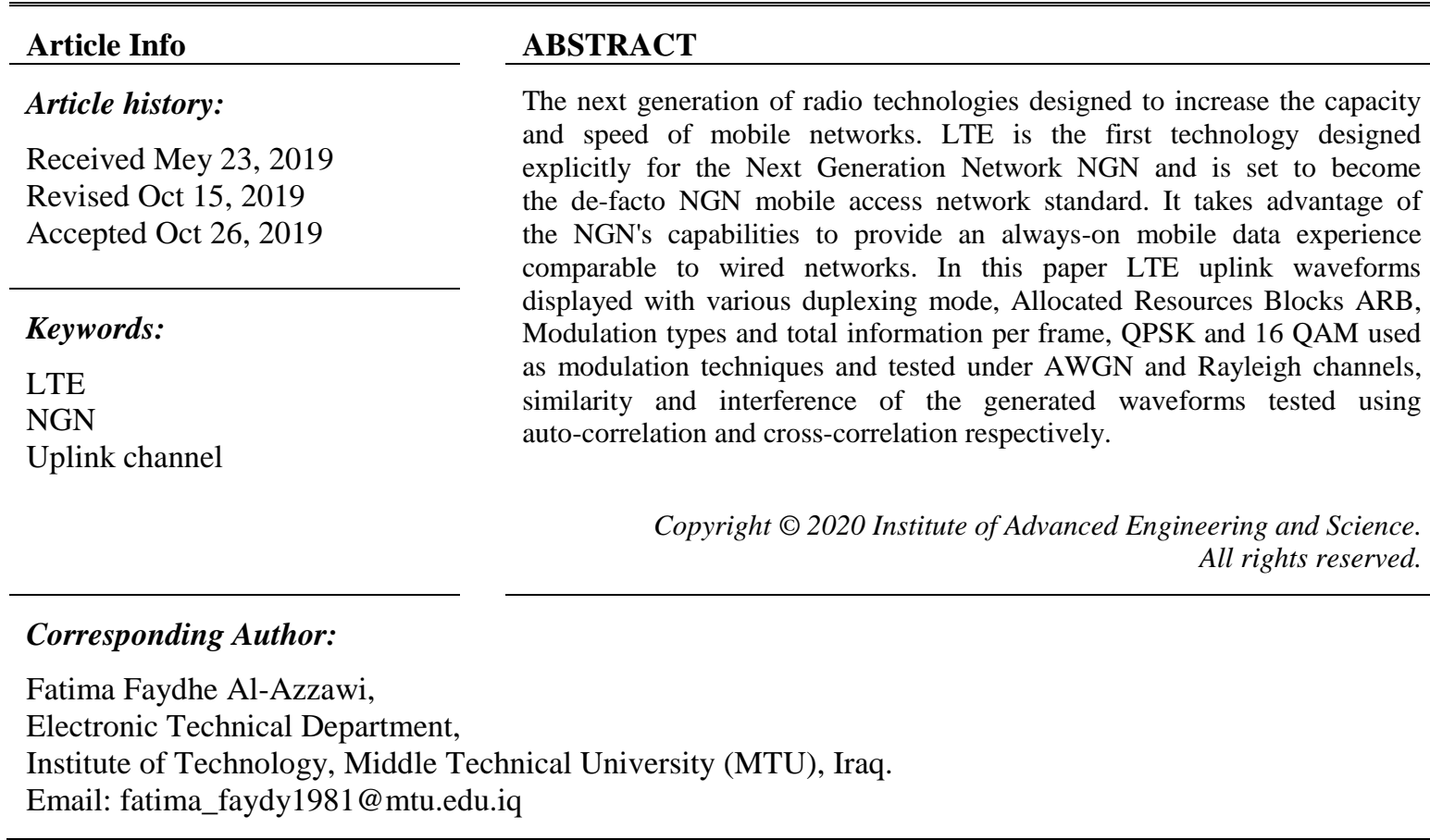

\section{INTRODUCTION}

To provide multiple services to customer, operators have built multiple networks such as fixed telephone networks, cable TV networks, cellular telephone networks and data networks. The Next Generation Network (NGN) provides all of these functions using a flat all-IP core that interconnects multiple access technologies and provides a consistent and reliable user-experience regardless of the access method. The NGN core will provide Quality of Service (QoS) support and a wide variety of applications and services. The NGN access network will provide mobility and routing management and ensure that the core sees the mobile networks simply as another IP network. Mobile handover between access types will be seamless as the IP access network controls security, authentication, and billing for the multiple access technologies. LTE is the first technology designed explicitly for the NGN and is set to become the de-facto NGN mobile access network standard. It takes advantage of the NGN's capabilities to provide an always-on mobile data experience comparable to wired networks $[1,2]$.

LTE supports peak data rates of up to $100 \mathrm{Mbps}$ on the downlink and $50 \mathrm{Mbps}$ on the uplink when using a $20 \mathrm{MHz}$ channel bandwidth, a single transmit antenna at the user equipment (UE), and two receive antennas at the Base Station. LTE generates three to four times the throughput on the downlink and two to three times the throughput on the Uplink relative to 3GPP Release 6. LTE improves spectrum efficiency, also defined relative to Release 6. The downlink target is three to four times the spectral efficiency of High-Speed Downlink Packet Access (HSDPA) while the uplink target is a two to three-time improvement over High-Speed Uplink Packet Access (HSDPA) [3, 4]. LTE has flexible duplex methods. Both Frequency Division Duplex (FDD) and Time Division Duplex (TDD) are valid spectrum allocations and allow LTE to accommodate various channel bandwidths in the available spectrum [5-9]. 
In this paper LTE uplink waveforms displayed with various duplexing mode, Allocated Resources Blocks ARB, Modulation types and total information per frame, QPSK and 16 QAM used as modulation techniques and tested under AWGN and Rayleigh channels, similarity and interference of the generated waveforms tested using auto-correlation and cross-correlation respectively.

\section{LTE UPLINK TRANSMISSION}

Explaining research chronological, including research design, research procedure (in the form of algorithms, Pseudocode or other), how to test and data acquisition $[1,3,10]$. The description of the course of research should be supported references, so the explanation can be accepted scientifically [4, 10]. The LTE standard does not only need wider bandwidth but also a more advanced modulation technique to matching the high data rates of LTE transmission. Orthogonal Frequency Division Multiplexing (OFDM) is considered to be the optimum modulation technique to fulfill the downlink transmission requirement, the high Peak-to-Average Power Ratio (PAPR) property of OFDM makes it less favorable for the uplink transmission. Instead, the Single-Carrier FDMA technique is used for LTE uplink transmission [9, 11-15].

The main advantage of using SC-FDMA instead of OFDMA in LTE Uplink Transmission scheme is the LTE uplink is supposed to provide up to $86 \mathrm{Mbit} / \mathrm{s}$ data rate. To achieve this, a new multi-access technique should be used. OFDMA can provide very high bandwidth but it also produces high PAPR (Peakto-Average Power Ratio). Since in mobile terminals we use batteries there should be efficient uplink transmission. Single-Carrier Frequency Division Multiple Access (SC-FDMA) is an answer to overcome the high PAPR problem. SC-FDMA has a lower PAPR which means that it will not consume as much power, giving a longer battery life to the user terminal [15-20].

In addition to the low-PAPR ('single-carrier' property) inherent in SC-FDMA, it also has some other desired properties for a transmission scheme. SC-FDMA allows for the possibility for low-complexity but high-quality equalization in the frequency domain and it is also possible to have flexible bandwidth assignments with SC-FDMA [9]. Another benefit of SC-FDMA is the so-called" built-in" frequency diversity. Because SC-FDMA spread the information of one symbol through all the available subcarriers, so in case Figure 1. Comparison between OFDMA and SC-FDMA losing partial information on one (or even more) subcarriers due to deep fading does not necessarily lead to losing the information modulated in the symbol [20-25].

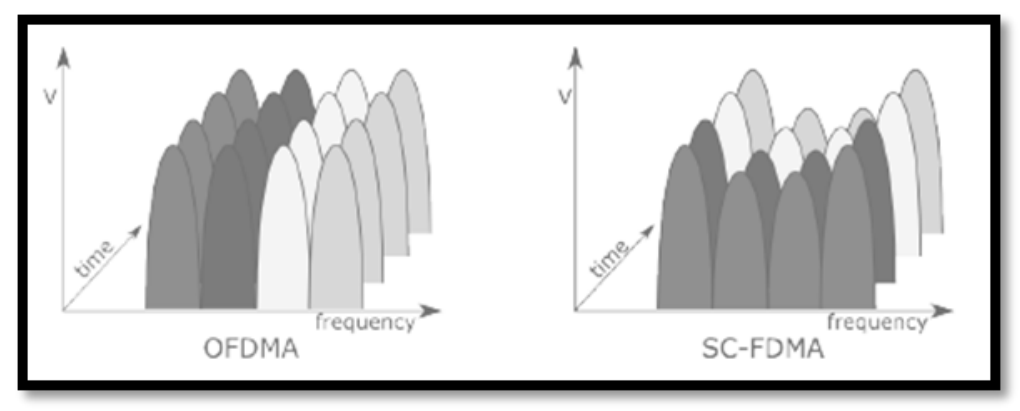

Figure 1. Comparison between OFDMA and SC-FDMA

The main components/elements of LTE that discussed in this paper are:

- Duplexing mode

- Resources Blocks

- Modulation types

LTE support both Frequency- and Time-Division-Duplex (FDD, TDD), meaning that in FDD different frequency bands are used for the downlink and uplink while in TDD downlink and uplink transmissions use the same frequency band but are done in separate time slots [2, 5]. Two frame types are defined for LTE: Type 1, used in Frequency Division Duplexing (FDD) as shown in Figure 2. Type 2, used in Time Division Duplexing (TDD) as shown in Figure 3. Type 1 frames consist of 20 slots with slot duration of $0.5 \mathrm{~ms}$. Type 2 frames contain two half frames. Depending on the switch period, at least one of the half frames contains a special subframe carrying three fields of switch information: Downlink Pilot Time Slot (DwPTS), Guard Period (GP) and Uplink Pilot Time Slot (UpPTS). If the switch time is $10 \mathrm{ms,}$, the switch 
information occurs only in subframe one. If the switch time is $5 \mathrm{~ms}$, the switch information occurs in both half frames, first in subframe one, and again in subframe six. Subframes 0 and 5 and DwPTS are always reserved for downlink transmission. UpPTS and the subframe immediately following UpPTS are reserved for uplink transmission. Other subframes can be uplink or downlink. One of the main advantages of using LTETDD systems is the ability to dynamically change bandwidth for uplink and downlink, depending on current needs, network load etc.

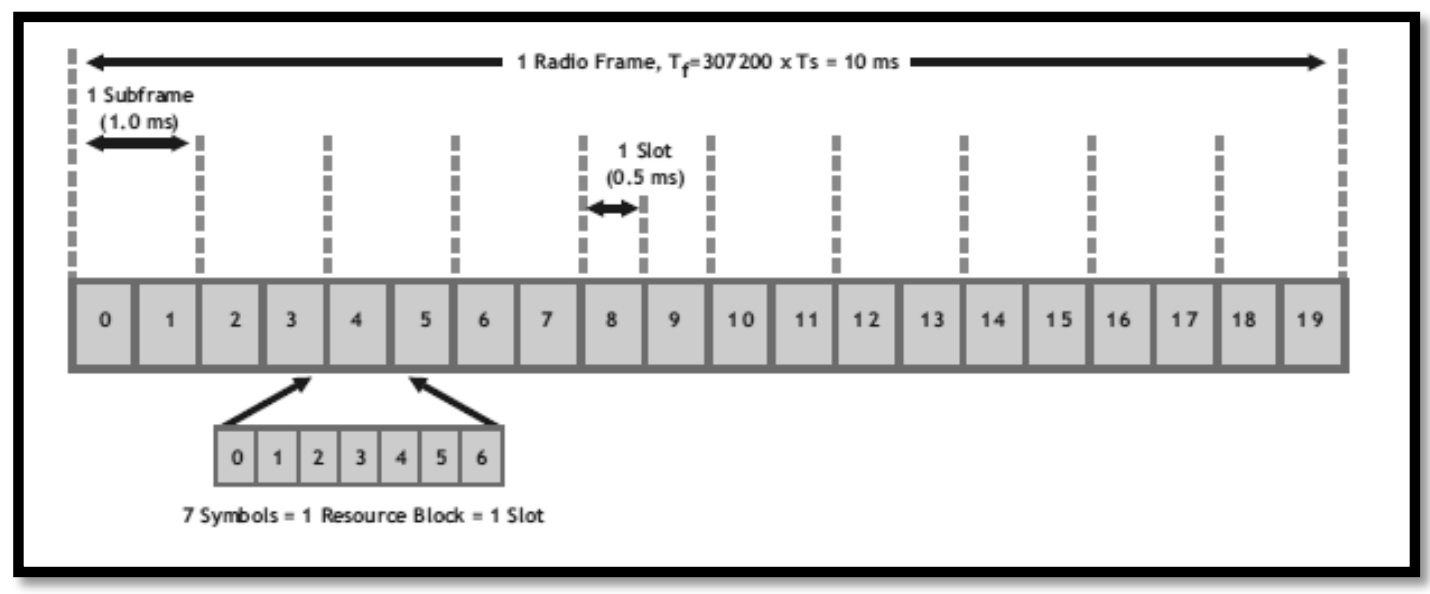

Figure 2. Type 1 Frame Type. Timing and symbol allocations shown for FDD with normal cyclic prefix (CP)

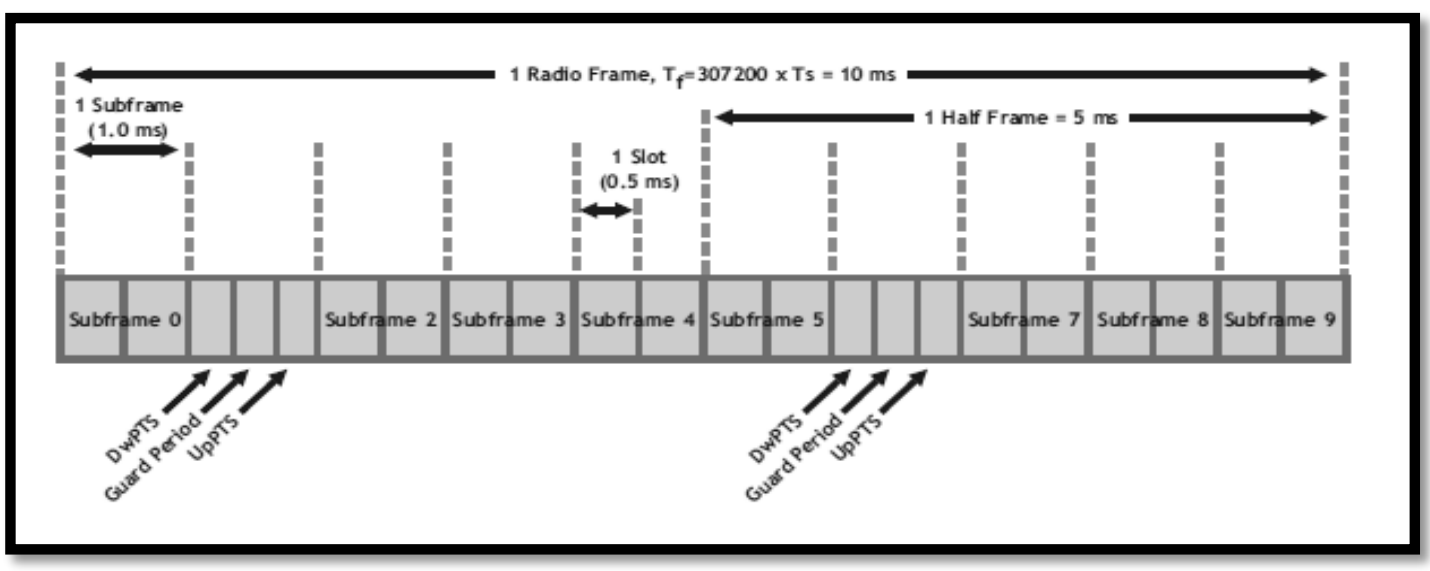

Figure 3. Type 2 frame type. Special fields are shown in subframes 1 and 6. Guard period separates the Downlink and Uplink. This TDD example represents a $5 \mathrm{~ms}$ switch point. A $10 \mathrm{~ms}$ switch point would not have the special fields in subframe 6

LTE resource block: In LTE, the time and frequency resources of the available bandwidth are divided into smaller blocks to support multiuser configuration and improve overall system efficiency. the available bandwidth is divided into number of orthogonal frequencies with a spacing of $\Delta \mathrm{f}=15 \mathrm{KHz}$ called subcarriers [11, 12]. This subcarrier spacing of $15 \mathrm{KHz}$ helps keeping Inter Carrier Interference (ICI) to the lower level even the mobile user is moving with high speed and causing high Doppler shifts in the frequency.

A resource block $(\mathrm{RB})$ or sub-frame is formed of a length $1 \mathrm{~ms}$ using 12 subcarriers and 12 or 14 OFDM symbols (depending on the length of Cyclic Prefix (CP)). Furthermore, the RB is subdivided into two slots of $0.5 \mathrm{~ms}$ each containing 6 or 7 OFDM symbols over 12 subcarriers. Such fine granularity of the time and frequency resources helps network to assign one or more RBs to different active users simultaneously depending upon the channel conditions and other factors. These building blocks are grouped together to form the radio resources $[13,14]$.

Duplexing mode, ARB and modulation approaches parameters affection on ... (Fatima Faydhe Al-Azzawi) 
In SC-FDMA as shown in Figure 4, the data is mapped into signal constellation according to the QPSK, 16-QAM, or 64-QAM modulation, depending upon the channel conditions similarly as in OFDMA. Whereas, the QPSK/QAM symbols do not directly modulate the subcarriers; these symbols pass through a serial to parallel converter followed by a DFT block that produce discrete frequency domain representation of the QPSK/QAM symbols. Pulse shaping is followed by DFT element, but it is optional and sometimes needs to shape the output signal from DFT. If pulse shaping is active then in the actual signal, bandwidth extension occurs. The Discrete Fourier symbols from the output of DFT block are then mapped with the subcarriers in subcarrier mapping block. After mapping the frequency domain; the modulated subcarriers pass through IDFT for time domain conversion. The rest of transmitter operation is similar as OFDMA $[8,15,16]$.

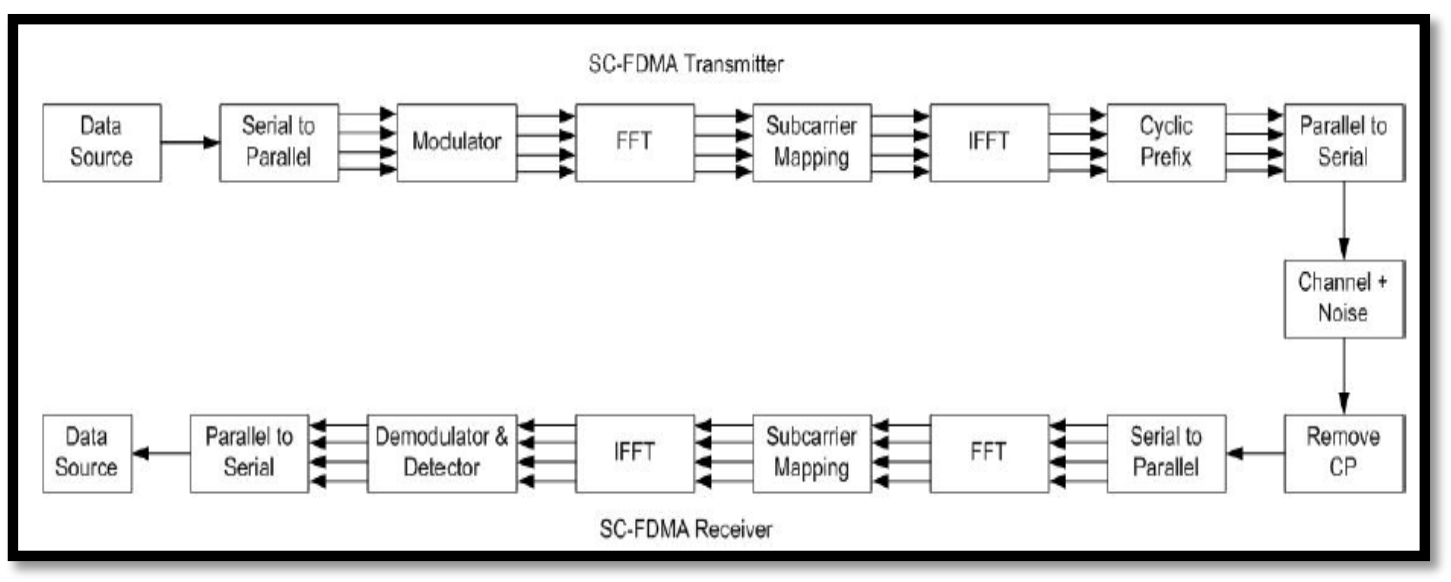

Figure 4. SC-FDMA system block diagram

\section{PRACTICAL RESULTS}

After implementing LTE uplink in MATLAB programing language, the proposed system testes under FDD and TDD duplexing mode, multi rate of resources Blocks, modulation type used of QPSK and 16 QAM leading of various number of information per frame as follows:

- Duplex mode Frequency Division Duplex (FDD), QPSK modulation type, allocated resource blocks 6, 15,25 , total information per frame $6000,15440,22160$ bits respectively.

From Figure 5 as number of resources block increased the band width increased so the total information per frame increased.

- Duplex mode Frequency Division Duplex (FDD), 16 QAM modulation type, allocated resource blocks $3,15,25$, total information per frame $23440,59920,99120$ bits respectively.

With FDD duplexing mode and 16 QAM the results shoes same increasing parentage in bandwidth as RB increased with using QPSK modulation as shown in Figure 6, total information per frame will increased with 16 QAM modulation.

- Duplex mode Frequency Division Duplex (TDD), QPSK modulation type, allocated resource blocks 6, 15,25 , total information per frame $2400,6177,8464$ bits respectively.

With TDD duplexing mode of Figure 7 if there is comparison with Figure 5, the results shoes same percentage in bandwidth increasing as ARB increased but number of total information per frame will decreased to 2400,6177, 8464 respectively with TDD wile with FDD 6000,15440, 22160 as number of AEB increased.

- Duplex mode Frequency Division Duplex (TDD), 16 QAM modulation type, allocated resource blocks 3, 15, 25, total information per frame 9376, 23968, 39648 bits respectively.

With TDD duplexing mode of Figure 8 as compared with Figure 7, 16 QAM increased number of total information per frame over QPSK, while comparison with Figure 6, FDD mod increases number of total information per frame over TDD as number ARB increased in addition to bandwidth that increased with QPSK, 16 QAM, FDD and TDD as ARB increased. 

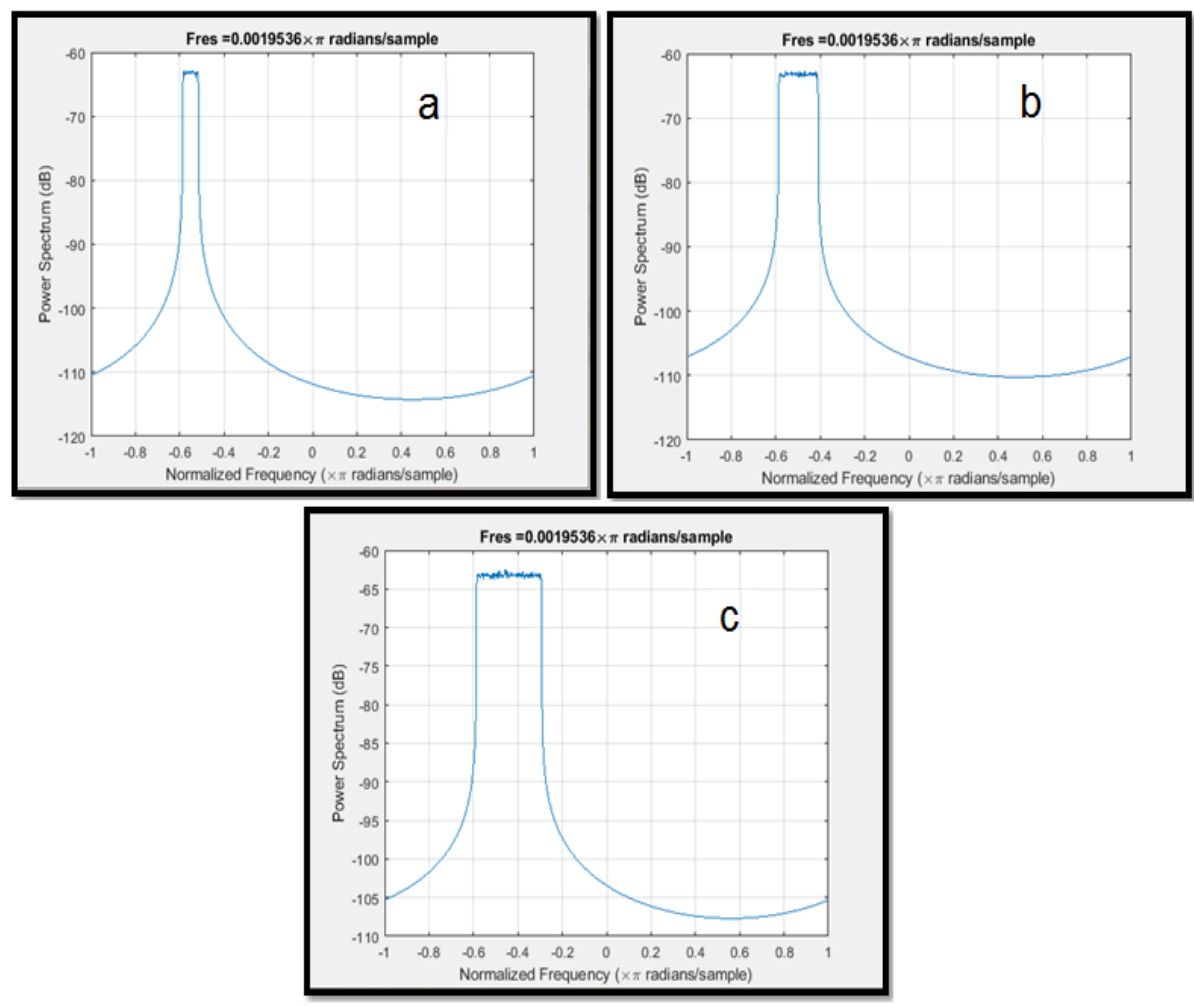

Figure 5. LTE uplink RMC wave form with QPSK modulation and FDD duplex mode, (a) RB 6, (b) RB 15, (c) RB 25
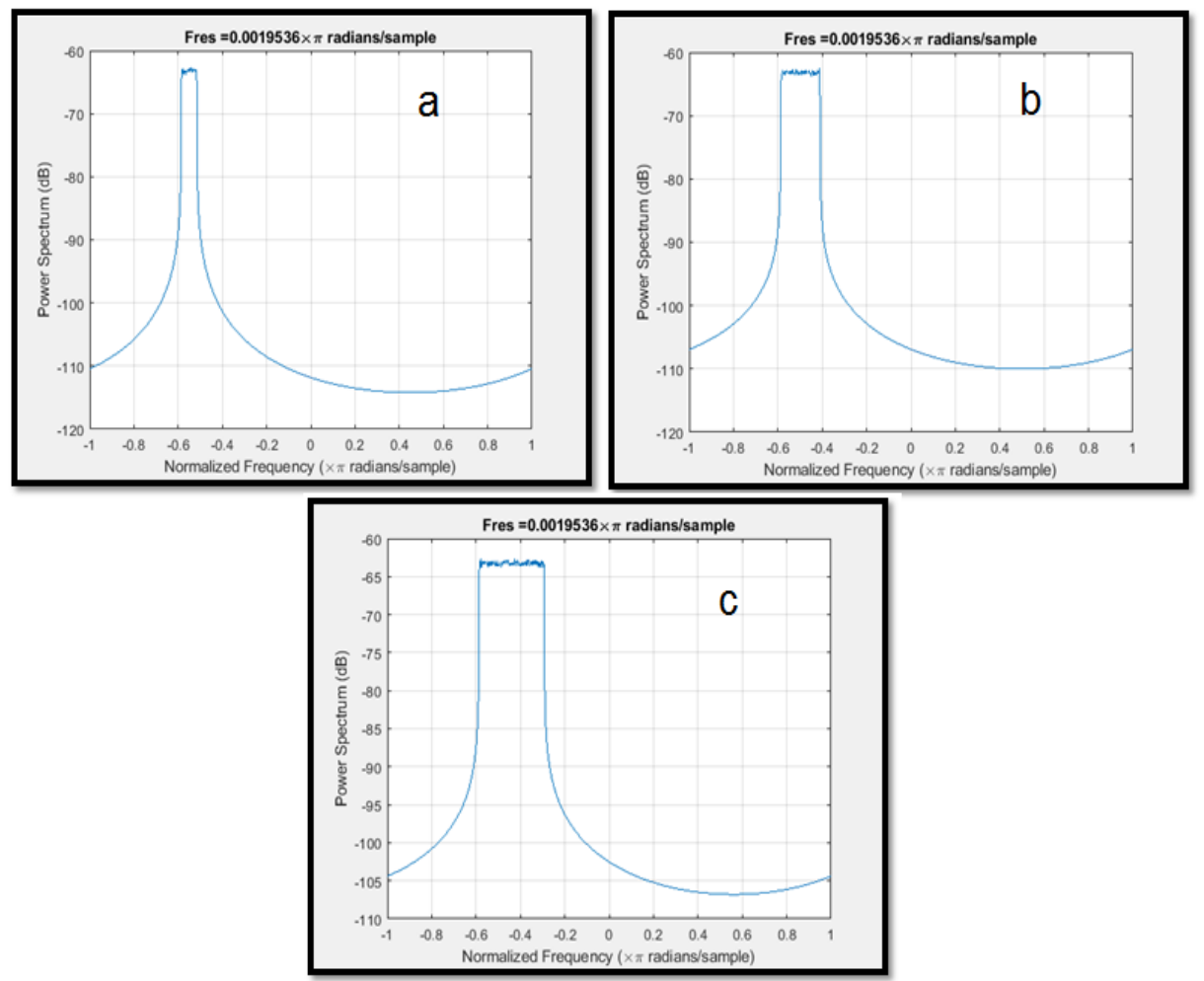

Figure 6. LTE uplink RMC wave form with 16 QAM modulation and FDD duplex mode, (a) RB 6, (b) RB 15, (c) RB 25 

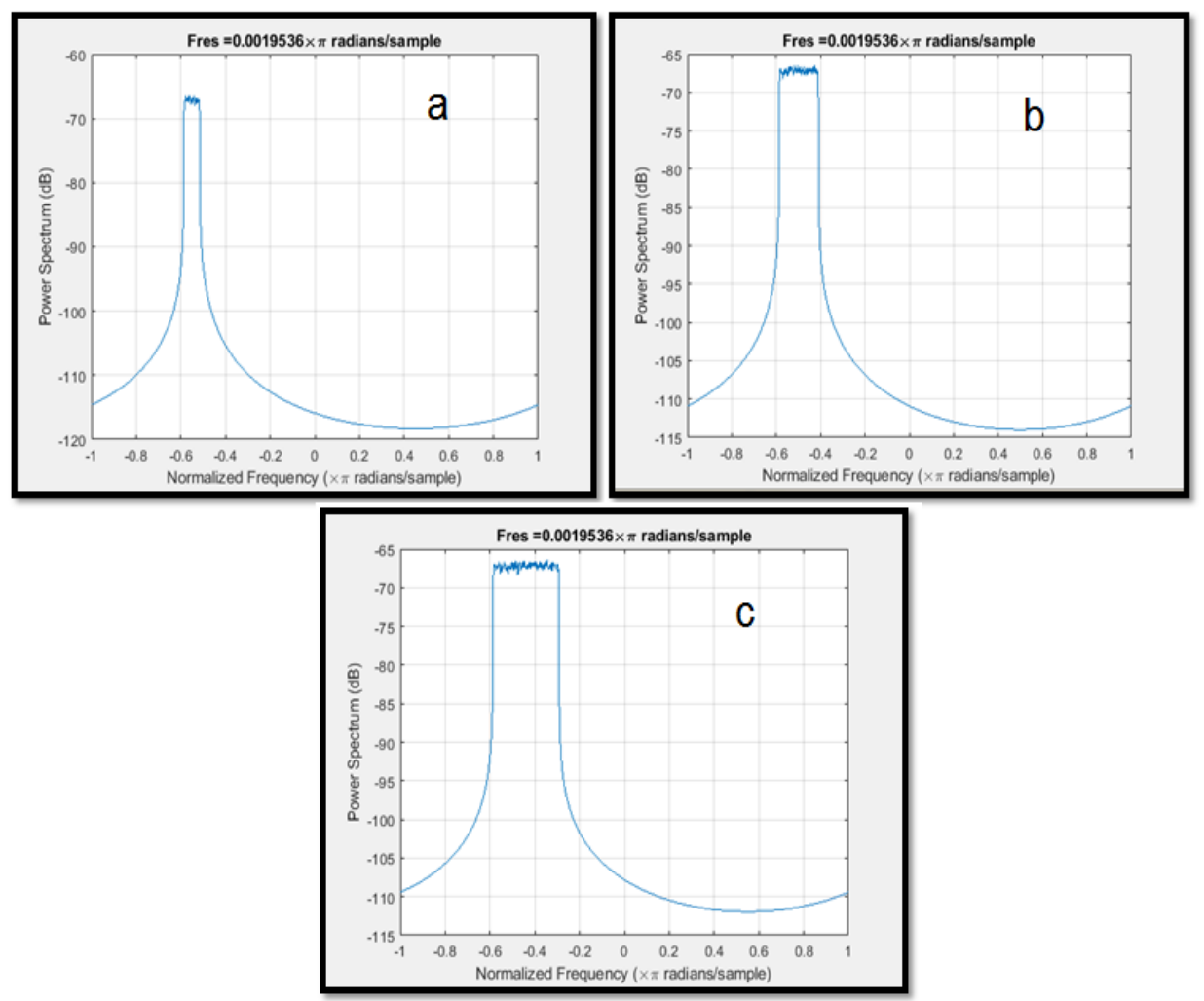

Figure 7. LTE uplink RMC wave form with QPSK modulation and TDD duplex mode, (a) RB 6, (b) RB 15 , (c) RB 25
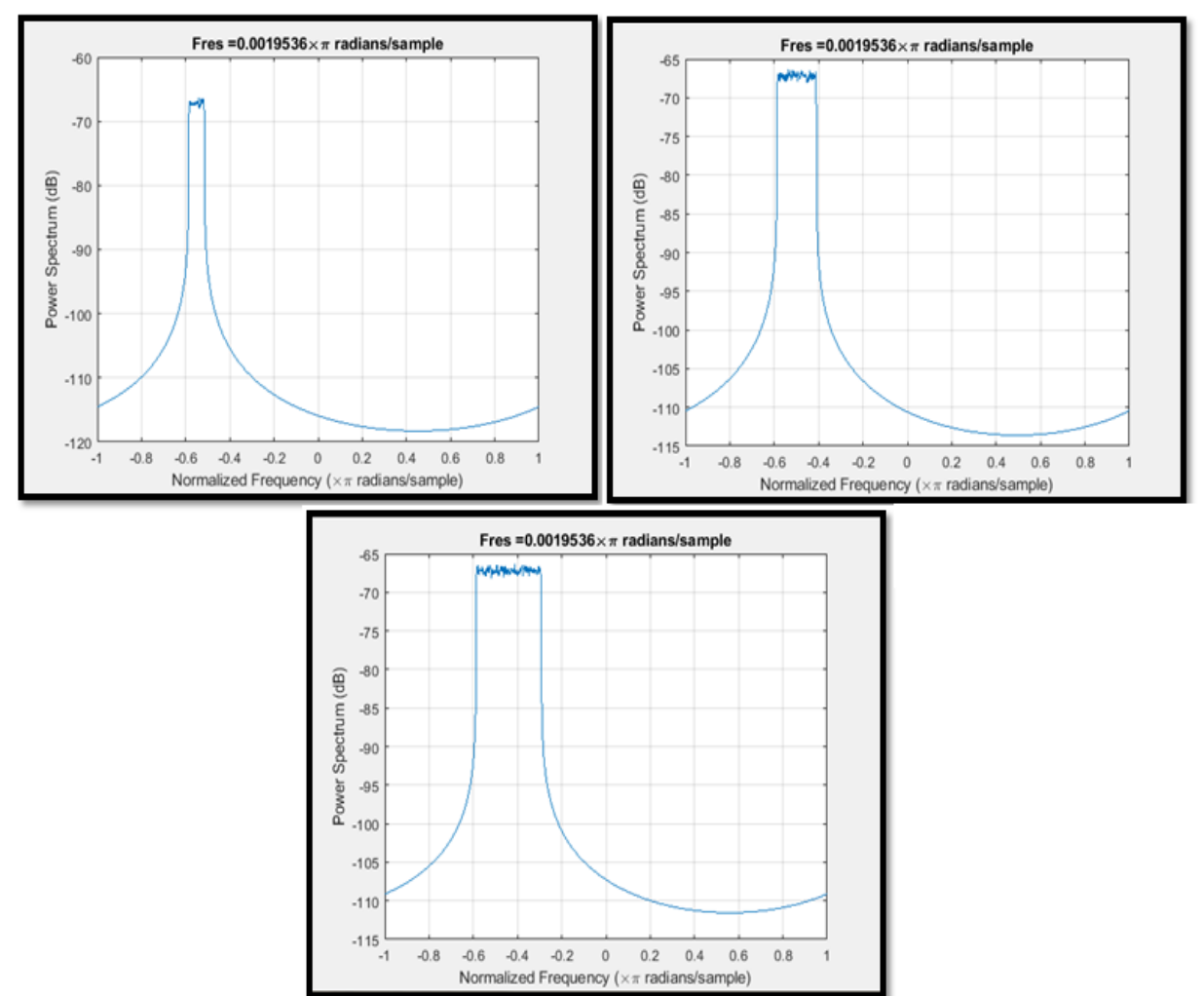

Figure 8. LTE uplink RMC wave form with 16 QAM modulation and TDD duplex mode, a. RB 6, b. RB 15, c. RB 25 
- Testing under AWGN and Rayleigh channels.

QPSK and 16 QAM tested under AWGN channel from Figure 9 the results show butter BER performance with QPSK under AWGN channel with gain $4 \mathrm{~dB}$ of $\mathrm{E}_{\mathrm{b}} / \mathrm{N}_{\mathrm{o}}$ in $10^{-8} \mathrm{BER}$ while under Rayleigh channel the gain in $\mathrm{E}_{\mathrm{b}} / \mathrm{N}_{\mathrm{o}}$ is $3 \mathrm{~dB}$ at $10^{-3}$ BER with QPSK modulation.

- Similarity test

LTE uplink with QPSK and 16 QAM waveforms examined using autocorrelation function in order to test the similarity of the wave forms, Figure 10 and Figure 11 shows the autocorrelation function of LTE uplink with QPSK and 16 QAM respectively with 6, 15, 25 allocated resources blocks, the results shows as number of allocated resources blocks increase the similarity decreased and the signal reaches the inequality.

- Crosstalk test

LTE uplink with QPSK and 16 QAM tested with cross correlation function in order to test the crosstalk or interference between signals as illustrated in Figure 12, from the results the cross correlation test shows butter performance as number of allocated resources block increased.

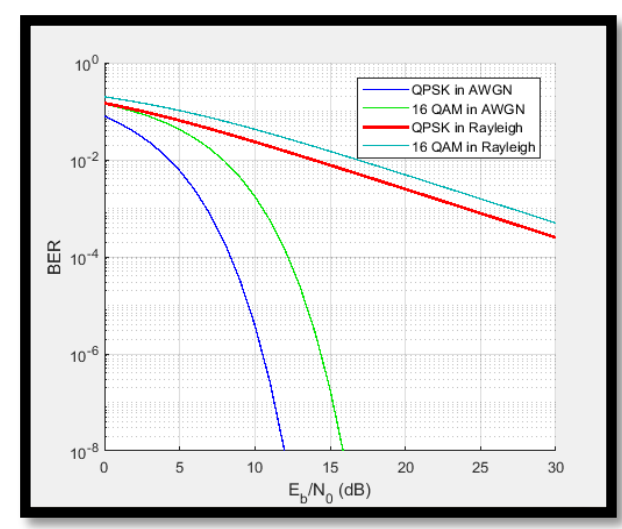

Figure 9. QPSK and 16 QAM under AWGN and Rayleigh fading channel

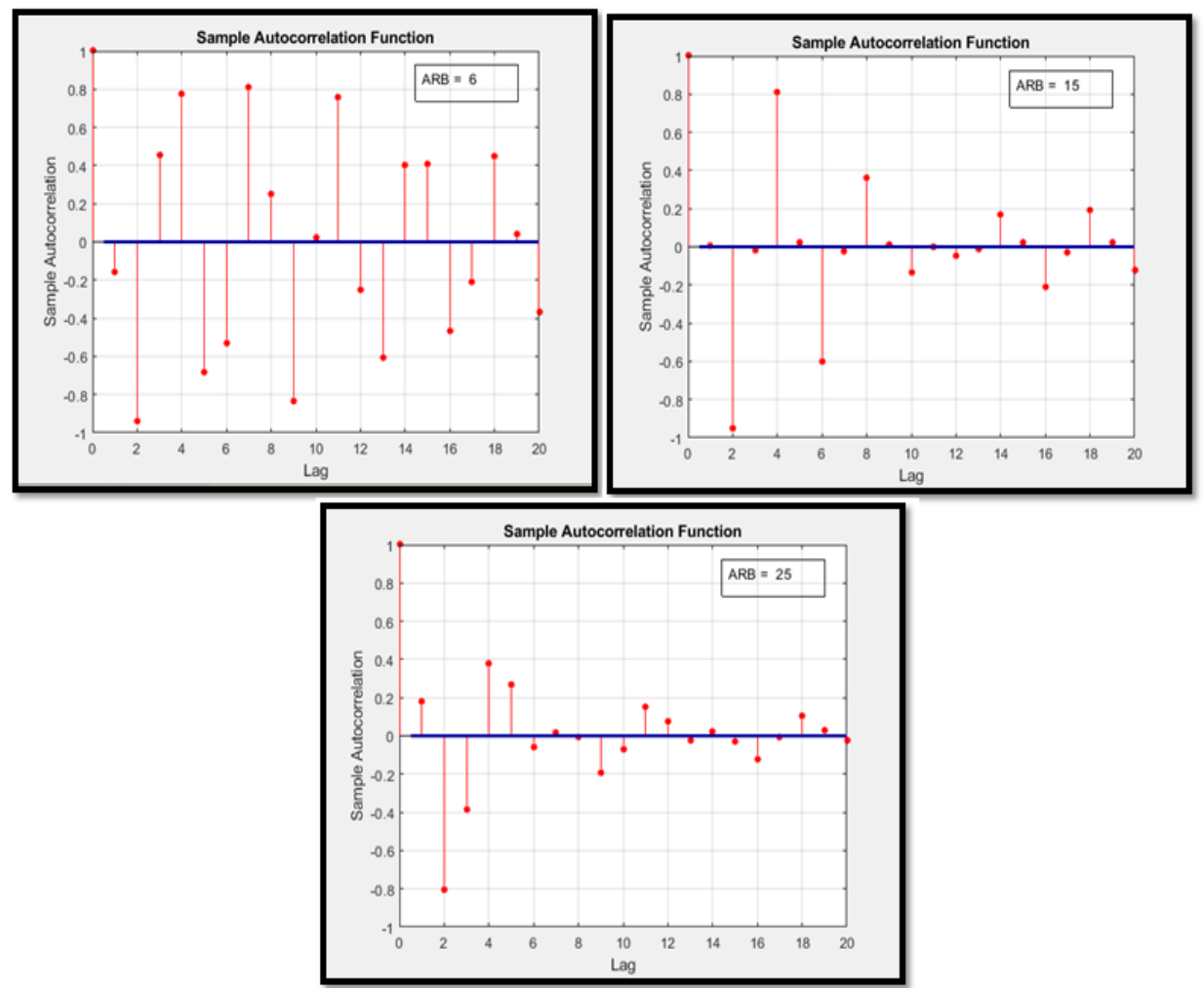

Figure 10. Autocorrelation test of LTE uplink waveform with QPSK modulation using various allocated resources blocks AEB

Duplexing mode, ARB and modulation approaches parameters affection on... (Fatima Faydhe Al-Azzawi) 

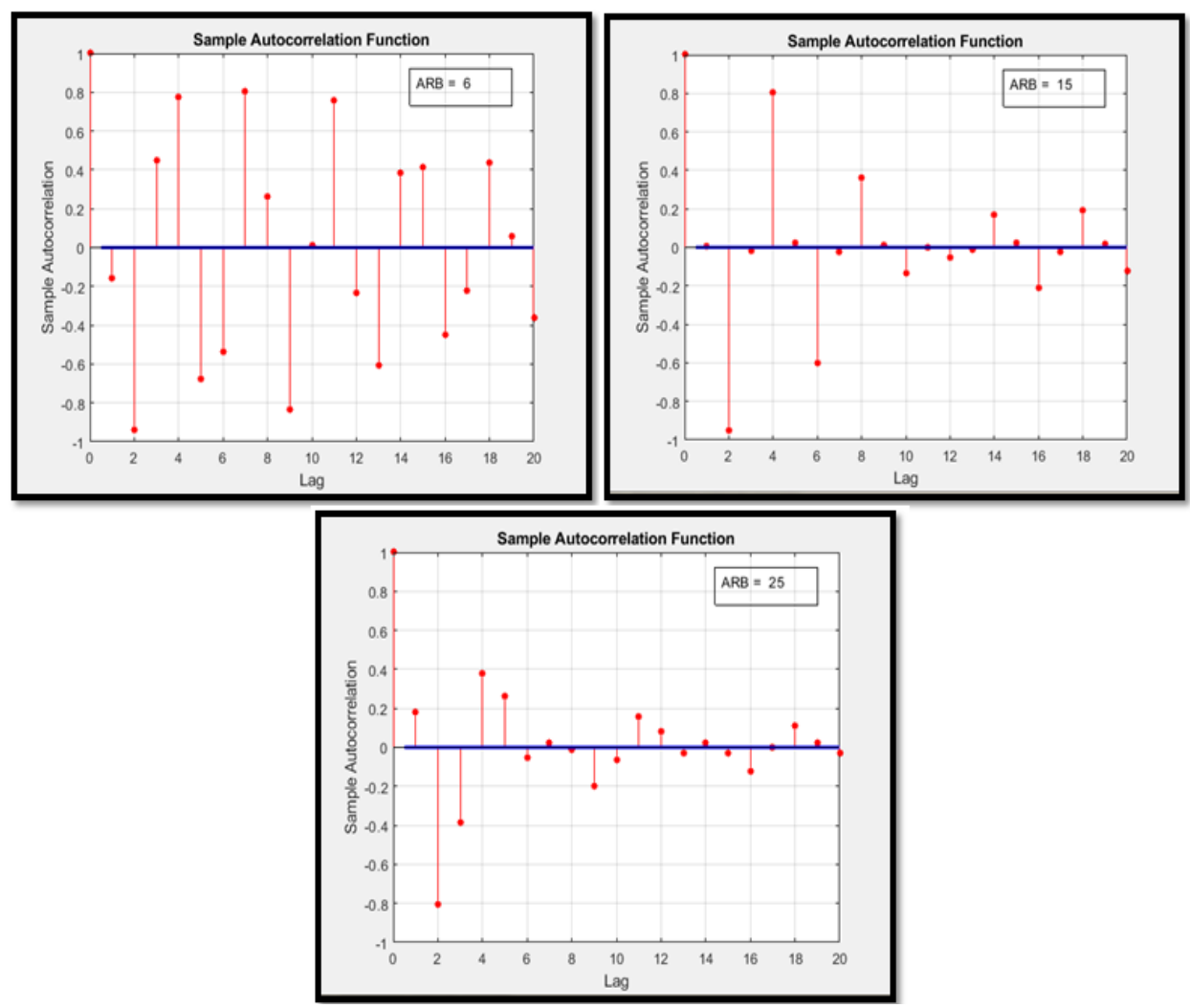

Figure 11. Autocorrelation test of LTE uplink waveform with 16 QAM modulation using various ARB
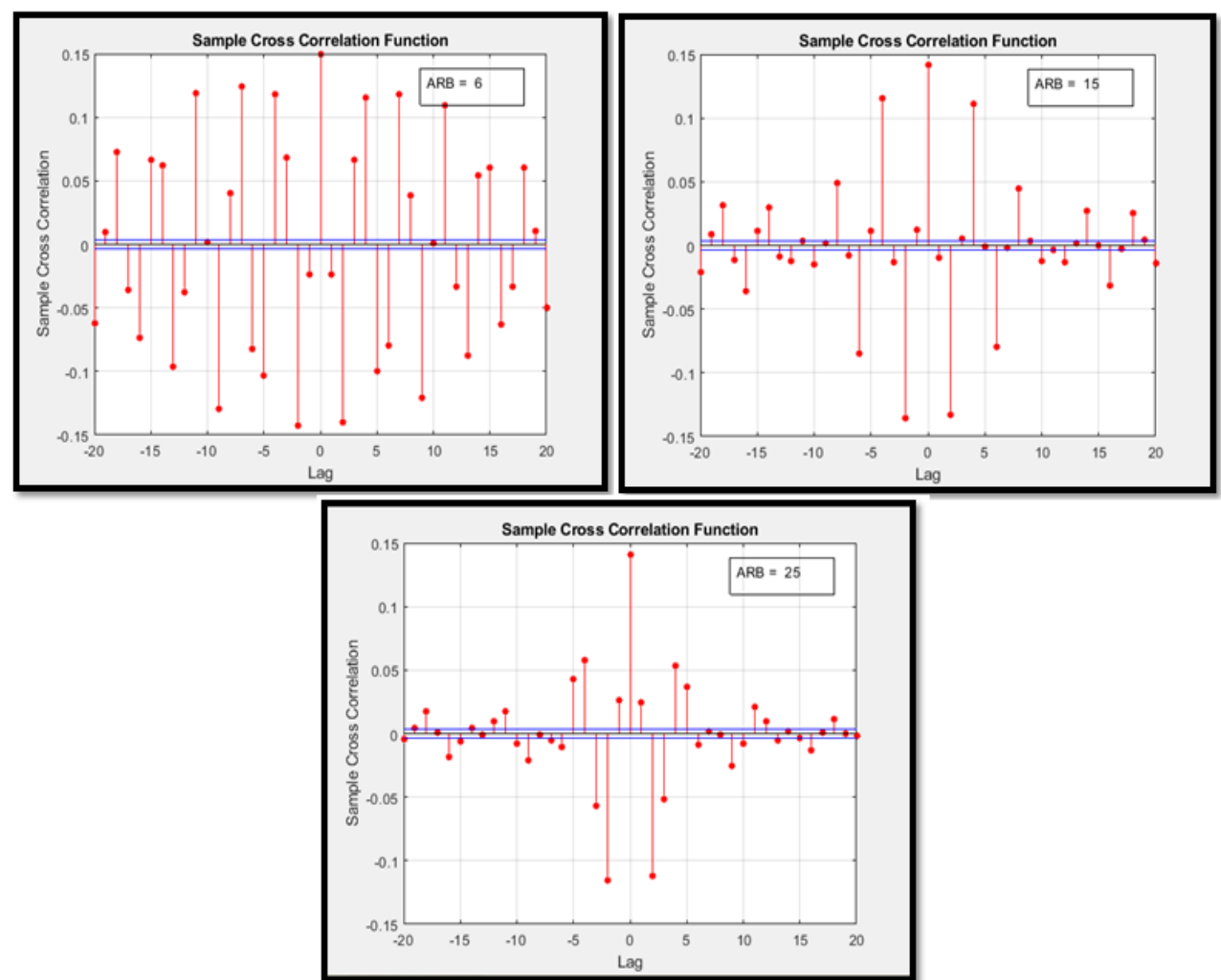

Figure 12. Cross correlation test of LTE uplink waveform between 16 QAM and QPSK modulation using various ARB 


\section{CONCLUSION}

In this paper LTE uplink proposed for the next generation of radio technologies and designed to increase the capacity and speed of mobile networks, where Duplexing mode, Resources Blocks, Modulation types of the LTE uplink is the parameters that effect on the practical results, LTE has flexible duplex methods. Both Frequency Division Duplex (FDD) and Time Division Duplex (TDD) are valid spectrum allocations and allow LTE to accommodate various channel bandwidths in the available spectrum. In LTE, the time and frequency resources of the available bandwidth are divided into smaller blocks to support multiuser configuration and improve overall system efficiency, as number of resources block increased the band width increased so the total information per frame increased, QPSK and 16 QAM modulation approaches used in the LTE uplink and tested under AWGN, Rayleigh channels from the results QPSK shows butter performance in term of BER with gain $4 \mathrm{~dB}$ and $3 \mathrm{~dB}$ in the tested channel respectively, similarity and crosstalk interference examined using auto-correlation and cross-correlation respectively, as number of ARB increased the performance of LTE uplink wave form is improved. Finally, the results of the parameters that examined in this paper which are Duplexing mode, Resources Blocks, Modulation types in the LTE uplink prove that the proposed system is the next generation of radio technologies.

\section{REFERENCES}

[1] G. Vijayalakshmy and G. Sivaradje, "Interworking of WLAN-LTE for next generation wireless networks," International Conference on Information Communication and Embedded Systems (ICICES2014), Chennai, pp. 1-6, 2014.

[2] Dan Jones (16 October 2012). "Defining 4G: What the Heck Is LTE TDD?," Light Reading. Retrieved 9 January 2014.

[3] Sidak, Gregory (2016). "What Aggregate Royalty Do Manufacturers of Mobile Phones Pay to License StandardEssential Patents" (PDF). The Criterion Journal on Innovation. Retrieved January 19, 2017.

[4] Galetovic, Alexander; Haber, Stephen; Zaretzki, Lew, "A New Dataset on Mobile Phone Patent License Royalties". Stanford University: Hoover Institution. Retrieved January 23, 2017.

[5] Ian Poole. "LTE-FDD, TDD, TD-LTE Duplex Schemes". Radio-electronics.com. Retrieved 9 January 2014.

[6] Hikmei Sari Cristina Ciochina. A review of ofdma and single-carrier fdma and some recent results. Advances in Electronics and Telecommunications, 1(1), April 2010.

[7] T. Hwang, C. Yang, G. Wu, S. Li and G. Ye Li, "OFDM and Its Wireless Applications: A Survey," in IEEE Transactions on Vehicular Technology, vol. 58, no. 4, pp. 1673-1694, May 2009.

[8] Guowang Miao, Jens Zander, Ki Won Sung, and Ben Slimane, "Fundamentals of Mobile Data Networks", Cambridge University Press, 2016.

[9] J. Skold P. Beming E. Dahlman, S. Parkvall. 3G Evolution: HSPA and LTE for Mobile Broadband. Academic Press, UK, 2nd edition, 2008.

[10] Sajal K. Das, "Mobile Terminal Receiver Design: LTE and LTE-Advanced", John Wiley \& Sons, April 2016.

[11] S. K. Jha, R. Rokaya, A. Bhagat, A. R. Khan and L. Aryal, "LTE Network: Coverage and Capacity Planning - 4G Cellular Network Planning around Banepa," 2017 International Conference on Networking and Network Applications (NaNA), Kathmandu, pp. 180-185, 2017.

[12] Chen, Qunhui (September 2011). "Evolution and Deployment of VoLTE" (PDF). Huawei Communicate Magazine (61). Archived from the original (PDF) on 2011-11-08..

[13] D. Astely, E. Dahlman, A. Furuskär, Y. Jading, M. Lindström and S. Parkvall, "LTE: the evolution of mobile broadband," in IEEE Communications Magazine, vol. 47, no. 4, pp. 44-51, April 2009.

[14] Fatime faydhe al-azzawi," LTE RF receiver modeling and each part testing with MATLAB simulink", Indonesian Journal of Electrical Engineering and Computer Science, Vol. 14, No. 3, pp. 1251 1257, June 2019.

[15] Zainab faydhe , Fatima faydhe, sada faydhe," M-PSK system performance in multi-channel environments with Matlab Simulink models", Journal of Engineering and Applied Sciences, 14(20):7509-7516, 2019.

[16] Fatima faydhe, faeza abas, Zainab faydhe, "Performance Comparison between DPSK and OQPSK modulation approaches in multi environments channels with Matlab Simulink models', Wasit Journal of Engineering Sciences, v(7), No.(1), 2019.

[17] Dr. Mohammed Aboud Kadhim, Baha Ali Nasir Fatima, Faydhe AL-Azzawi," Design and Development of HiperLAN/2 Physical Layer Model Based Wavelet Signals" Journal of Information Engineering and Applications, Vol.4, No.6, 2014

[18] Dr. Mohammed Aboud Kadhim,Dr.Ahamed Rashied,Fatima Faydhe AL-Azzawi," Design and Performance of LTE 3GPP Baseband Transceiver Based Wavelet Signals for Different Channel Estimation Algorithms", Journal of Information Engineering and Applications, Vol.4, No.9, 2014.

[19] Dr. Emad Shehab Ahmed, Fatima, Faydhe AL-Azzawi,” Frequency Hopped-Orthogonal Frequency Division Multiple Access (FH-OFDMA) System Performance with Extending Quadratic Congruence (EQC) Hoping Code patterns", Journal of Engineering and Development, Vol. 12, No. 1, March (2008).

[20] Fatima, Faydhe AL-Azzawi ,Dr. Emad Shehab Ahmed," Effect of multi-tone jamming on FH-OFDMA system with orthogonal hopping patterns", Effect of multi-tone jamming on FH-OFDMA system with orthogonal hopping patterns, IEEE GCC Conference (GCC), 2006. 
[21] Fatima Faydhe AL-Azawi, Zainab Faydh AL-Azawi, Rafed shaker AL- Fartosy, "Design and Simulation of Pulse Code Modulation Multiplexing (PCMM) and De-Multiplexing Technique in MATLAB"International Journal of Innovative Technology and Exploring Engineering (IJITEE) Volume-3, Issue-10, March 2014.

[22] Fatima Faydhe AL-Azzawi," Minimization the Effect of Different Channel Model on QAM and PAM Systems in Term of Bit Error Rate", International Journal of Soft Computing and Engineering (IJSCE), Volume-4, Issue-1, March 2014.

[23] Agilent Technologies, LTE and the Evolution to 4G Wireless: Design and Measurement Challenges, John Wiley \& Sons, 2009.

[24] H. Ekström, A. Furuskär, J. Karlsson, M. Meyer, S. Parkvall, J. Torsner, and M. Wahlqvist, "Technical Solutions for the 3G Long-Term Evolution," IEEE Commun. Mag., vol. 44, no. 3, pp. 38-45, March 2006.

[25] Y. Zhou, Z. Lei and S. H. Wong, Evaluation of Mobility Performance in 3GPP Heterogeneous Networks 2014 IEEE 79th Vehicular Technology Conference (VTC Spring), Seoul, pp. 1-5, 2014. 